\title{
Long noncoding RNA papillary thyroid carcinoma susceptibility candidate 3 (PTCSC3) inhibits proliferation and invasion of glioma cells by suppressing the $\mathrm{Wnt} / \beta$ - catenin signaling pathway
}

Shujun Xia, Ri Ji and Weiwei Zhan*

\begin{abstract}
Background: The dysregulation of long noncoding RNAs (IncRNAs) has been identified in a variety of cancers. An increasing number of studies have found the critical role of IncRNAs in the regulation of cellular processes, such as proliferation, invasion and differentiation. Long noncoding RNA papillary thyroid carcinoma susceptibility candidate 3 (PTCSC3) is a novel IncRNA that was primarily detected in papillary thyroid carcinoma. However, the biological function and molecular mechanism of IncRNA PTCSC3 in glioma are still unknown.

Methods: The expression level of IncRNA PTCSC3 in human microglia and glioma cell lines was examined using quantitative real-time polymerase chain reaction (qRT-PCR). The influence of IncRNA PTCSC3 on cell proliferation were studied using the cell counting kit-8, and cell cycle and apoptosis were analyzed by flow cytometry assays. The migration and invasion abilities were investigated by transwell and wound healing assays. The target genes of IncRNA PTCSC3 were explored by qRT-PCR, immunofluorescence and western blot.

Results: LnCRNA PTCSC3 was significantly downregulated in glioma cell lines. The overexpression of IncRNA PTCSC3 suppressed proliferation and induced apoptosis in U87 and U251 cells. Additionally, the overexpression of IncRNA PTCSC3 inhibited the migration and invasion of U87 and U251 cells. Moreover, InCRNA PTCSC3 inhibited the epithelialmesenchymal transition of U87 cells. The study also demonstrated that LRP6, as a receptor of the Wnt/ $\beta$-catenin pathway, was a target of IncRNA PTCSC3. By evaluating the expression levels of Axin1, active $\beta$-catenin, c-myc, and cyclin D1, the study indicated that InCRNA PTCSC3 inhibited the activation of the Wnt/ $\beta$-cateninpathway through targeting LRP6.
\end{abstract}

Conclusions: LnCRNA PTCSC3 inhibits the proliferation and migration of glioma cells and suppresses Wnt/B-catenin signaling pathway by targeting LRP6. LnCRNA PTCSC3 is a potential therapeutic target for treatment of glioma.

Keywords: LncRNA PTCSC3, Glioma, Proliferation, Invasion, EMT, Wnt signal

\footnotetext{
* Correspondence: shanghairuijinus@163.com

Ultrasound Department, Rui Jin Hospital Shanghai Jiao Tong University

School of Medicine, 197 Rui Jin Er Road, Huang Pu District, Shanghai Zip

code: 200025, People's Republic of China
} 


\section{Background}

Glioma appears to be one of the most common types of primary brain tumors in adults $[1,2]$. Characterized by rapid progression, patients with glioma are most likely diagnosed at advanced stages, and the prognosis remains poor [3], posing a large threat to human health. Although some advances in comprehensive treatment as well as early diagnosis have been made, only a few patients have experienced the expected effects when translated to the clinic $[4,5]$. Thus, it is imperative to explore the mechanisms concerning glioma formation and progression and to establish diagnostic and therapeutic targets for optimized management of glioma.

Long noncoding RNAs (lncRNAs) are defined as nonprotein coding transcripts longer than 200 nucleotides. Recently, IncRNAs have gained much attention in the field of molecular biology. Increasing evidence indicates that lncRNAs are involved in diverse biological processes, including cell proliferation, differentiation, apoptosis, development and immune responses [6, 7]. Papillary thyroid carcinoma susceptibility candidate 3 (PTCSC3) is an intergenic long noncoding RNA gene (lincRNA) located at 14q.13.3, which was newly identified as thyroid specific [8]. Subsequently, lncRNA PTCSC3 was reported to be a tumor suppressor in thyroid cancer [9], and the mechanism study demonstrated that IncRNA PTCSC3 reduced cell motility and invasiveness by downregulating the S100A4 pathway [10]. However, little is known about the role of lncRNA PTCSC3 in other malignancies.

Our study was performed to assess the expression of lncRNA PTCSC3 in glioma cells and to evaluate its role and mechanism in tumor cell proliferation, invasion and migration. This is the first time that lncRNA PTCSC3 has been assessed in glioma. We assessed the expression level of lncRNA PTCSC3 in human microglia and glioma cell lines. Additionally, we demonstrated that lncRNA PTCSC3 overexpression suppressed proliferation, migration and invasion and inhibited the epithelialmesenchymal transition (EMT) by suppressing the Wnt/ $\beta$-catenin signaling pathway in glioma.

\section{Methods}

\section{Cell culture}

Several glioma cell lines (U87, U251, SHG44 \& SHG139) were purchased from the Cell Bank Type Culture Collection of the Chinese Academy of Sciences (Shanghai, China). Human microglia was purchased from the Scinencell Research Laboratories (Carlsbad, CA, USA). Human astrocyte was purchased from Lonza (Basel, Switzerland) and cultured in AGM $^{\mathrm{m}}$ Astrocyte Growth Medium. Other cells were cultured in DMEM (Gibco, Carlsbad, CA, USA) supplemented with $10 \%$ of fetal bovine serum (Gibco) and 1\% of penicillin-streptomycin at $37^{\circ} \mathrm{C}, 5 \% \mathrm{CO}_{2}$ humidified atmosphere.
RNA extraction and quantitative reverse transcriptasepolymerase chain reaction (qRT-PCR)

Total RNA was extracted from cell lines with TRIzol reagent (Invitrogen, Carlsbad, CA, USA) and the concentration was measured by nanodrop spectrophotometer. One microgram of total RNA was reversely transcribed into first-strand cDNA using a Reverse Transcription Kit (Takara, Dalian, China). PCR was performed in VIIA7 system (Applied Biosystems, California, USA) with SYBR ${ }^{\circ}$ Premix Ex Taq ${ }^{\mathrm{TM}}$ II Kit (Takara, Dalian, China). GAPDH was used as an internal control. Gene primers are listed in Table 1. Comparison Ct $(2-\Delta \Delta \mathrm{Ct})$ method was used to analyze the data.

Table 1 Sequence of primers used for qRT-PCR

\begin{tabular}{|c|c|c|}
\hline Gene & & Sequence $\left(5^{\prime}-3^{\prime}\right)$ \\
\hline \multirow[t]{2}{*}{ IncRNA PTCSC3 } & Forward Primer & GGCTTGAACAATCTTCCCACCTT \\
\hline & Reverse Primer & TTGGCAACACCCTCACAGACAC \\
\hline \multirow[t]{2}{*}{ MMP1 } & Forward Primer & AAAATTACACGCCAGATTTGCC \\
\hline & Reverse Primer & GGTGTGACATTACTCCAGAGTTG \\
\hline \multirow[t]{2}{*}{ MMP2 } & Forward Primer & CCCACTGCGGTITTCTCGAAT \\
\hline & Reverse Primer & CAAAGGGGTATCCATCGCCAT \\
\hline \multirow[t]{2}{*}{ MMP9 } & Forward Primer & AGACCTGGGCAGATTCCAAAC \\
\hline & Reverse Primer & CGGCAAGTCTTCCGAGTAGT \\
\hline \multirow[t]{2}{*}{ MMP13 } & Forward Primer & ACTGAGAGGCTCCGAGAAATG \\
\hline & Reverse Primer & GAACCCCGCATCTTGGCTT \\
\hline \multirow[t]{2}{*}{ E-cadherin } & Forward Primer & CGAGAGCTACACGTTCACGG \\
\hline & Reverse Primer & GGGTGTCGAGGGAAAAATAGG \\
\hline \multirow[t]{2}{*}{ Fibronectin } & Forward Primer & CGGTGGCTGTCAGTCAAAG \\
\hline & Reverse Primer & AAACCTCGGCTTCCTCCATAA \\
\hline \multirow[t]{2}{*}{ Snail } & Forward Primer & TCGGAAGCCTAACTACAGCGA \\
\hline & Reverse Primer & AGATGAGCATTGGCAGCGAG \\
\hline \multirow[t]{2}{*}{ ZEB1 } & Forward Primer & GATGATGAATGCGAGTCAGATGC \\
\hline & Reverse Primer & ACAGCAGTGTCTTGTTGTTGT \\
\hline \multirow[t]{2}{*}{ FZD8 } & Forward Primer & ATCGGCTACAACTACACCTACA \\
\hline & Reverse Primer & GTACATGCTGCACAGGAAGAA \\
\hline \multirow[t]{2}{*}{ LRP6 } & Forward Primer & TाTATGCAAACAGACGGGACTT \\
\hline & Reverse Primer & GCCTCCAACTACAATCGTAGC \\
\hline \multirow[t]{2}{*}{ Axin1 } & Forward Primer & GACCTGGGGTATGAGCCTGA \\
\hline & Reverse Primer & GGCTTATCCCATCTTGGTCATC \\
\hline \multirow[t]{2}{*}{ C-myc } & Forward Primer & GGCTCCTGGCAAAAGGTCA \\
\hline & Reverse Primer & CTGCGTAGTTGTGCTGATGT \\
\hline \multirow[t]{2}{*}{ Cyclin D1 } & Forward Primer & TGGAGCCCGTGAAAAAGAGC \\
\hline & Reverse Primer & TCTCCTTCATCTTAGAGGCCAC \\
\hline \multirow[t]{2}{*}{ GAPDH } & Forward Primer & AAGGTGAAGGTCGGAGTCAAC \\
\hline & Reverse Primer & GGGGTCATTGATGGCAACAATA \\
\hline
\end{tabular}




\section{Lentivirus transfection}

The lncRNA PTCSC3 was ligated into pLVX-IRES-puro to construct the lncRNA PTCSC3 overexpression plasmid. The pRUF-IRES-puro-PTCSC3 and pLVX-IRES-puro constructs were transfected into the HEK293T viral packaging cell line together with psPAX2 and pMD2.G plasmids. Forty-eight hours after transfection, the supernatant was collected and used to infect the cells.

\section{CCK-8 assay}

U87 and U251 were selected as the appropriate cell lines for gain-of-function experiments. The two cell lines were seeded into 96-well plates at a density of $1 \times 10^{4}$ cells/ well $48 \mathrm{~h}$ after transfection and cultured at $37{ }^{\circ} \mathrm{C}, 5 \%$ $\mathrm{CO}_{2}$ incubator. The viabilities of cells were assessed with the cell counting kit-8 (CCK-8; Dojindo Molecular Technologies, Japan) at four time points $(12,24,48$ \& $72 \mathrm{~h}$ ). The absorbance was detected by a TECAN infinite M200 plate reader at $450 \mathrm{~nm}$.

\section{Colony formation assay}

For colony formation assay, 500 cells were seeded in each well of a six-well plate $48 \mathrm{~h}$ after transfection and cultured at $37{ }^{\circ} \mathrm{C}, 5 \% \mathrm{CO} 2$ incubator with the medium being changed every 2 days. On the 7th day, the cultural media was removed, and cells were washed twice with PBS. After that, colonies were fixed in methanol for $20 \mathrm{~min}$ and stained with $1 \%$ crystal violet (Sigma) for $30 \mathrm{~min}$, and then washed. Colonies were counted and photographed.

\section{Flow cytometry assay}

Fourty-eight hours after transfection, U87 and U251 cells were collected for cell cycle and apoptosis analysis. The cell cycle analysis was performed using the Cell Cycle Analysis Kit (Beyotime biotechnology, Jiangsu, China) according to the manufacturer's instructions. The apoptosis assay was performed using the Alexa Fluor ${ }^{\circ}$ 488 Annexin V/Dead Cell Apoptosis Kit (Thermo Fisher Scientific, Waltham, MA, USA). The cells were detached by trypsinase, centrifuged at $1000 \mathrm{~g}$ for $5 \mathrm{~min}$ and then resuspended in $100 \mu \mathrm{L}$ of binding buffer with $5 \mu \mathrm{L}$ of Annexin v. After that, $1 \mu \mathrm{L}$ of propidium iodide was added and mixed, followed by 15 min' incubation in dark. Subsequently, $400 \mu \mathrm{L}$ of binding buffer was added to resuspend the cells, which were analyzed by Gallios Flow Cytometry (Beckman Coulter, USA). The experiments were repeated triple times.

\section{Transwell assay}

Cell invasion abilities were performed with transwell assay using transwell chambers with $8-\mu \mathrm{m}$ pores (Costar, Corning, NY, USA). In invasion assay, the upper side of the insert membrane was coated with matrigel (BD
Biosciences, New Jersey, USA). A number of $1 \times 10^{4}$ cells in $200 \mu \mathrm{l}$ of serum-free medium were added into the upper chamber and $600 \mu \mathrm{l}$ of medium with $5 \%$ FBS as chemoattractant was added into the lower chamber. After $24 \mathrm{~h}$ ' incubation, the cells on the upper surface of the insert membrane were removed using cotton swab. The cells on the lower surface of the insert membrane were fixed in methanol for $20 \mathrm{~min}$ and then stained with $1 \%$ crystal violet for $30 \mathrm{~min}$. The number of cells on the lower surface of the membrane was calculated with a microscope and then photographed. All experiments were performed in triplicate.

\section{Scratch assay}

Cells were seeded in each well of 6-well plates and cultured until $100 \%$ confluence. A scratch was performed using pipette tips and then serum-free medium was replaced. The scratch closing procedure was observed in $24 \mathrm{~h}$ and images were taken after $24 \mathrm{~h}$ ' incubation.

\section{Western blot analysis}

Total protein was extracted from collected cells with RIPA buffer containing phenylmethanesulfonylfl uoride (PMSF). After a 30-min reaction on ice, the protein lysate was centrifuged at $13000 \mathrm{rpm}$ for $15 \mathrm{~min}$ and the supernatant was collected. The protein concentration was measured by the BCA Protein Assay Kit (Pierce, Illinois, USA). Ten microgram of each protein was subjected to $8 \%$ SDS-PAGE and transferred to PVDF membranes, which was then blocked with $5 \%$ BSA in TBST (TBS containing 0.1\% Tween-20) at room temperature for $1 \mathrm{~h}$ and incubated with the corresponding primary antibodies diluted in blocking buffer at $4{ }^{\circ} \mathrm{C}$ overnight. After washing with PBST (PBS containing $0.1 \%$ Tween), secondary antibodies was added for incubation at room temperature for $1 \mathrm{~h}$. The western blot images were captured using the LI-COR Odyssey Scanner. Primary antibodies recognizing human E-cadherin, Fibronectin, LRP6, Axin1, active catenin, Cmyc, Cyclin D1 (mouse mAb, 1:1000, Abcam, Cambridge, MA, USA) and GAPDH (rabbit mAb, 1:2000, Cell Signaling Technology, Danvers, MA, USA) were used.

\section{Immunofluorescence assay}

U87 cells were seeded in $35-\mathrm{mm}$ cell culture dishes (NEST Biotech, Wuxi, China). The cells were fixed with 4\% PFA in PBS for $20 \mathrm{~min}$ and permeabilized with $0.5 \%$ Triton X-100 for $5 \mathrm{~min}$ at room temperature. After washing in PBS, cells were blocked with 5\% BSA for $1 \mathrm{~h}$ and incubated with primary antibodies against E-Cadherin and Fibronectin (1:200, Abcam, Cambridge, MA, USA) overnight at $4{ }^{\circ} \mathrm{C}$, and then detected using Alexa fluor 546-conjugated anti-mouse IgG secondary antibodies, followed by a $2-\mathrm{mg} / \mathrm{mL}$ solution of 4', 6-diamidino-2- 
phenylindole (DAPI; Sigma-Aldrich, St. Louis, MO, USA) in PBS for nuclear staining. Cells were visualized under a confocal microscope (Leica, Solms, Germany).

\section{RNAi}

For RNAi-mediated knockdown experiment, lncRNA PTCSC3-specific siRNA and scramble negative control siRNA (ScrsiRNA) were purchased from GenePharma Co., Ltd (Shanghai, China). The sequences were as follow: IncRNA PTCSC3-siRNA, sense, 5' -AUCUUUUGCAUUA AUCUCCCU-3', antisense, 5'-GGAGAUUAAUGCAAA AGAUGG-3'; ScrsiRNA, sense, 5'-UUCUCCGAACGUG UCACGUTT-3', antisense, and 5'-ACGUGACACGUU CGGAGAATT-3'. U87 cells were transfected with 100 nM lncRNA PTCSC3 specific siRNA or scrsiRNA using Lipofectamine ${ }^{\mathrm{TM}} 2000$ (Thermo Fisher Scientific, Waltham, MA, USA) according to the manufacturer's protocol. The transfection efficiency was confirmed by qRT-PCR (See Additional file 1: Figure S1).

\section{Statistical analysis and quantification}

All statistical analyses were performed using Statistical Program for Social Sciences 19.0 software (SPSS, Chicago, IL, USA) and GraphPad Prism 5.0 (GraphPad Software, LaJolla, CA, USA). Data were presented as the means \pm SD. The significance of differences between groups was estimated by Student's $t$-test (two-sided, unpaired). $P<0.05$ was considered to indicate a statistically significant difference. All experiments were repeated at least three times.

\section{Results}

LncRNA PTCSC3 overexpression inhibits the proliferation and induces apoptosis in glioma cells

The expression level of lncRNA PTCSC3 in glioma was detected in human microglia cells, astrocyte and four glioma cell lines (U87, U251, SHG44 \& SHG139) by qRT-PCR. LncRNA PTCSC3 was downregulated in glioma cell lines compared with human microglia cells and astrocyte (Fig. 1). Among the four glioma cell lines, U87 had the lowest expression level of IncRNA PTCSC3 compared with U251, SHG44 and SHG139 (Fig. 1). U87 and U251 were selected to investigate the biological function of lncRNA PTCSC3 in glioma cells. As lncRNA PTCSC3 was downregulated in glioma cell lines, the gain-of-function experiment was performed using lentivirus vector in the U87 and U251 glioma cell lines. The transfection efficiency was determined by qRT-PCR after $72 \mathrm{~h}$ of transfection. As shown in Fig. 2a, the expression levels of IncRNA PTCSC3 in U87 and U251 cells transfected with the lncRNA PTCSC3 overexpression vector were increased by 189.34 -fold and 152.01-fold respectively, compared with control cells. In CCK8 assay, the cell viabilities of U87 and U251 cells

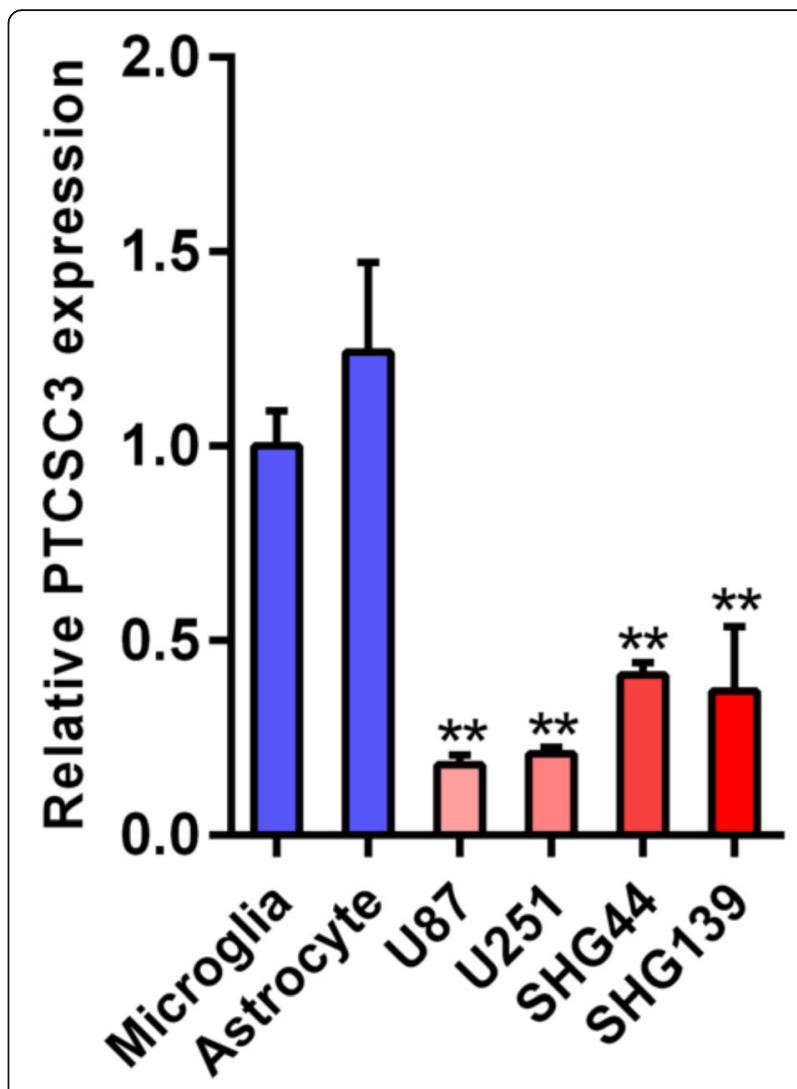

Fig. 1 Expression of IncRNA PTCSC3 in human microglia and glioma cell lines. qRT-PCR analysis of the expression levels of IncRNA PTCSC3 in human microglia, astrocyte and glioma cell lines (U87, U251, SHG44 \& SHG139). The values were normalized to GAPDH mRNA expression. Data are expressed as the means \pm S.D of three independent experiments. "**" indicates $P<0.01$

were determined at five time points $(0,12,24,48$, and $72 \mathrm{~h}$ ) after being transfected with the lncRNA PTCSC3 overexpression vector. The viability of the U87 and U251 cells decreased significantly at $48,72 \mathrm{~h}$ with overexpression of lncRNA PTCSC3 compared with the control (Fig. 2b, c). In cell cycle analysis, the proportions of cells in the G1 phase was significantly increased in U87 and U251 cells with lncRNA PTCSC3 overexpression compared with the control. Additionally, the proportion of cells in $\mathrm{S}$ and $\mathrm{G} 2 / \mathrm{M}$ phases were reduced in the lncRNA PTCSC3 overexpression group (Fig. 3d-f). In colony formation assay, there were fewer colonies in the lncRNA PTCSC3 overexpression group compared with the control (Fig. 3g, i). Moreover, the apoptosis assay showed that IncRNA PTCSC3 overexpression promoted apoptosis in U87 and U251 cells (Fig. 4a-c). Our result indicated that lncRNA PTCS3 overexpression inhibited proliferation by inducing cell cycle arrest and apoptosis in glioma cells as well as suppressing cell viability. 
A

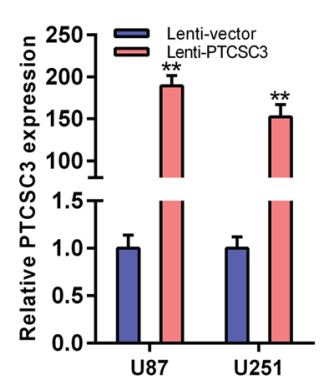

D
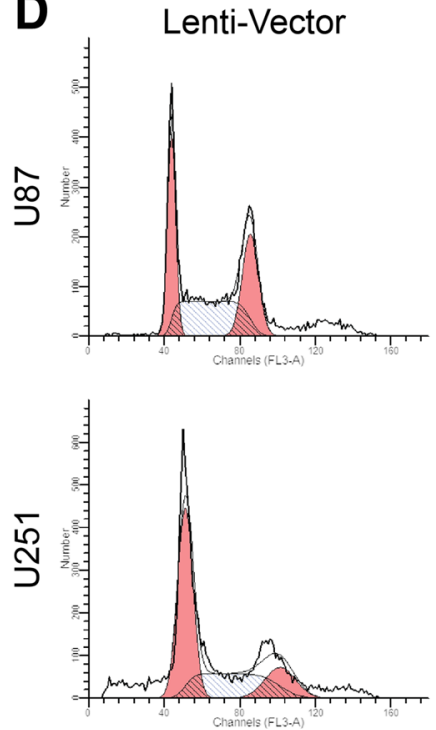

G
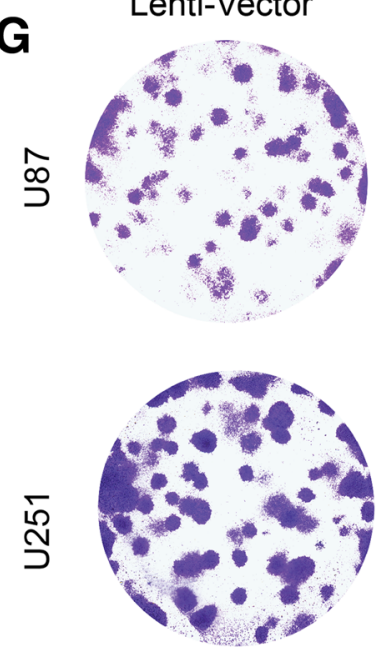

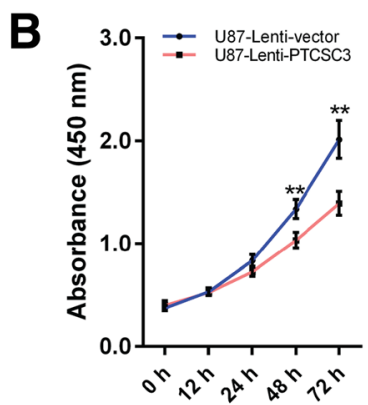

Lenti-PTCSC3

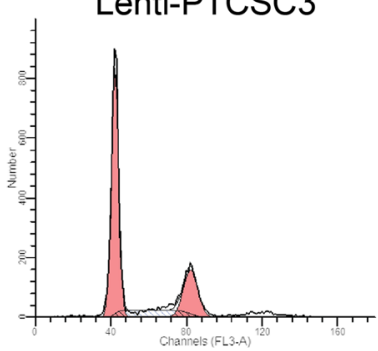

E
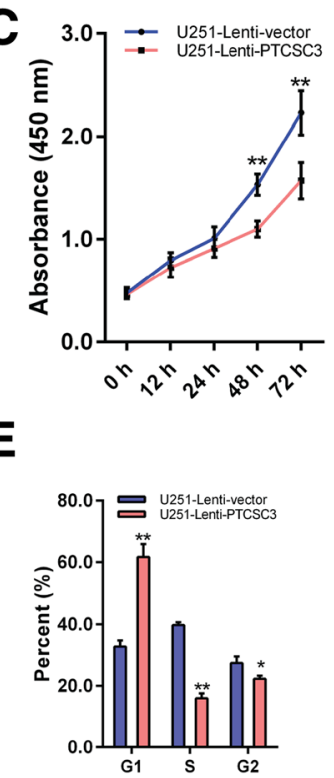

F

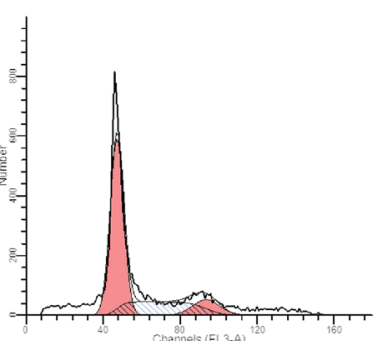

Lenti-PTCSC3

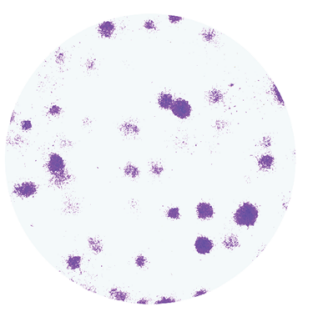

H
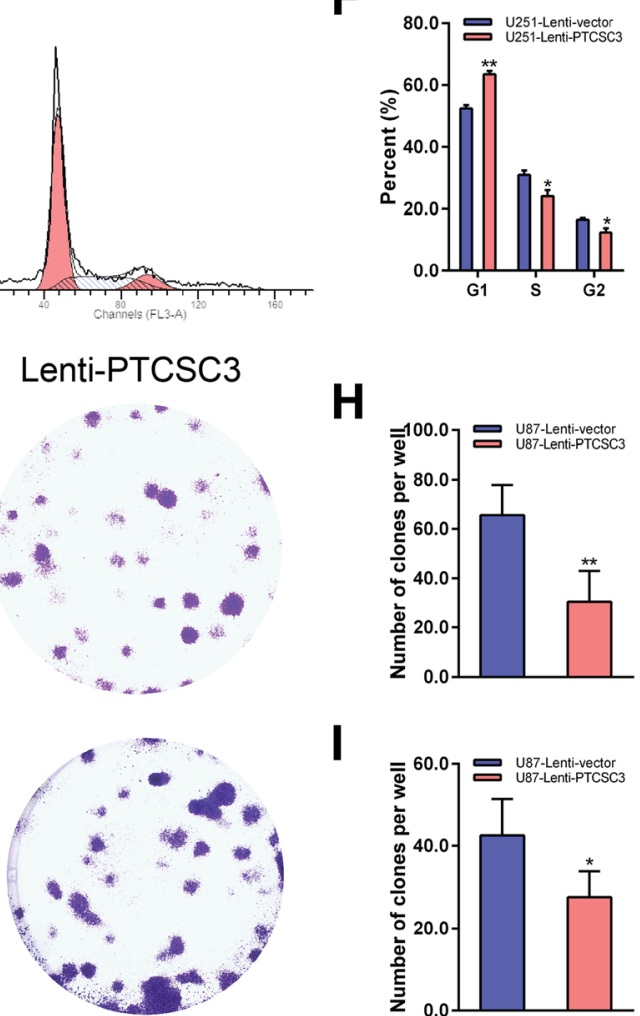

I

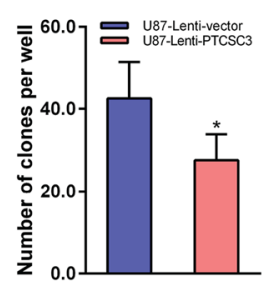

Fig. $\mathbf{2}$ LnCRNA PTCSC3 overexpression inhibits the proliferation of U87 and U251. a qRT-PCR analysis of IncRNA PTCSC3 expression levels in U87 and U251 infected with Lenti-Vector or Lenti-PTCSC3. CCK-8 assays were performed to determine U87 (b) and U251 (c) cell proliferation after $12 \mathrm{~h}, 24 \mathrm{~h}, 48 \mathrm{~h}$ and $72 \mathrm{~h}$ infected with Lenti-Vector or Lenti-PTCSC3. d Representative flow cytometry images of the cell cycle in U87 and U251 cells infected with Lenti-Vector or Lenti-PTCSC3. The cell cycle results quantified in of U87 (e) and U251 (f) cells are presented as the percentage of total cells. $\mathbf{g}$ Colony formation assay was performed to determine the proliferation of U87 and U251 cells infected with Lenti-Vector or LentiPTCSC3. The colonies were captured and counted. Colony formation assay results of U87 (h) and U251 (i) cells are presented as histograms. Data are expressed as the means \pm S.D of three independent experiments. " ${ }^{* \prime \prime}$ indicates $P<0.05$, "***" indicates $P<0.01$ 

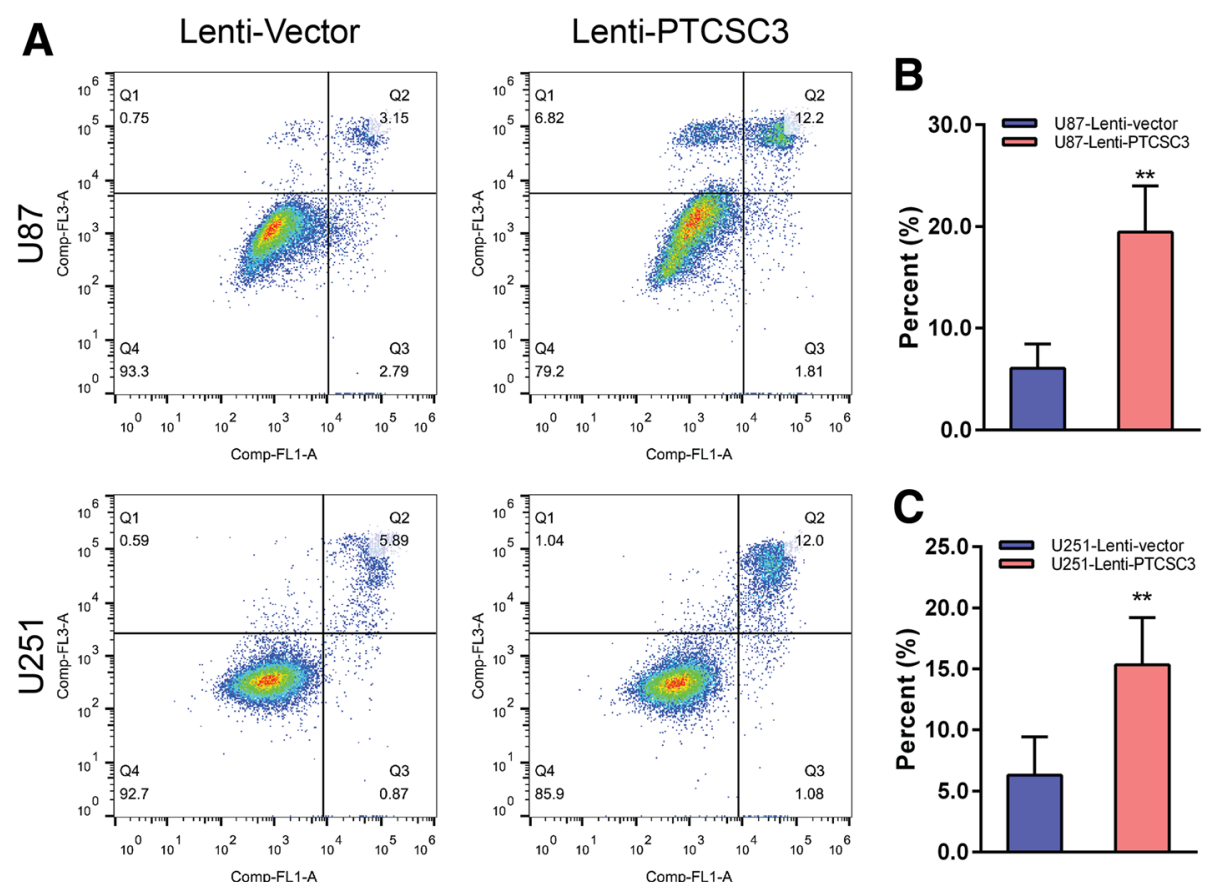

Fig. 3 LnCRNA PTCSC3 overexpression induces apoptosis in U87 and U251. a Representative flow cytometry images of apoptosis of U87 and U251 cells infected with Lenti-Vector or Lenti-PTCSC3. The percentage of apoptotic U87 (b) and U251 (c) cells is presented as histograms. Data are expressed as

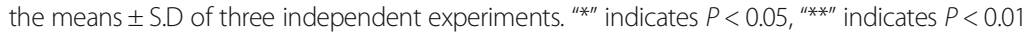

\section{LncRNA PTCSC3 overexpression inhibits migration and invasion in glioma cells}

Scratch test and transwell assay were used to evaluate the affect of lncRNA PTCSC3 on glioma cell migration and invasion respectively. The scratch test showed that lncRNA PTCSC3 overexpression remarkably inhibits U87 cell migration (Fig. 4a), which was quantified by the percentage of the wound area (Fig. 4c). The transwell invasion assay showed that overexpression of lncRNA PTCSC3 reduced the invasive capacity of U87 (Fig. 4b, d) and U251 cells (Fig. 4b, e). Further, the expression of matrix metalloproteinases (MMPs) in lncRNA PTCSC3 elevated cells were detected to investigate a potential mechanism for lncRNA PTCSC3 in the inhibition of migration and invasion, indicating that MMP2 and MMP9 expression were downregulated in IncRNA PTCSC3 overexpressed cells, while MMP1 and MMP13 remained unaffected (Fig. $4 \mathrm{f}-\mathrm{i}$ ). These data showed that lncRNA PTCS3 overexpression inhibited glioma cell migration and invasion via downregulating MMP2 and MMP9 expression.

\section{LncRNA PTCSC3 overexpression inhibits the epithelial- mesenchymal transition of U87 cells}

To investigate the affect of lncRNA PTCSC3 on the epithelial-mesenchymal transition of glioma cells, the study examined the mesenchymal markers and epithelial markers in U87 cells with or without lncRNA PTCSC3 overexpression. It showed that E-cadherin (epithelial marker) was upregulated (Fig. 5a) while Fibronectin, Snail and ZEB1 (mesenchymal markers) were downregulated in lncRNA PTCSC3 overexpressed cells (Fig. 5b-d). The function of lncRNA PTCSC3 increased the expression of E-cadherin and reduced the expression of Fibronectin was further confirmed by western blot (Fig. 5e and Additional file 2: Figure S2) and immunofluorenscence (Fig. 5f).

\section{LncRNA PTCSC3 overexpression inhibits the Wnt/B-catenin signaling pathway by suppressing LRP6}

The affect of lncRNA PTCSC3 on the Wnt/ $\beta$-catenin signaling pathway was investigated in U87 cell line. Data indicated that the expression of LRP6, C-myc and Cyclin D1 were downregulated and the expression of Axin1 was upregulated when lncRNA PTCSC3 was overexpressed, wherease the expression of FZD8 remained unchanged (Fig. 6a-e). Conversely, the expression of LRP6, C-myc and Cyclin D1 were induced while that of Axin1 was reduced when lncRNA PTCSC3 was knocked down, wherease FZD8 expression remained unchanged (Fig. 6a-e). In western blot analysis, lncRNA PTCSC3 overexpression downregulated LRP6 expression, subsequently suppressing the expression of active $\beta$-catenin, $\mathrm{C}$-myc and cyclin D1, while increasing Axin1 expression, and vice versa (Fig. $6 \mathrm{f}$ and Additional file 3: Figure S3). 

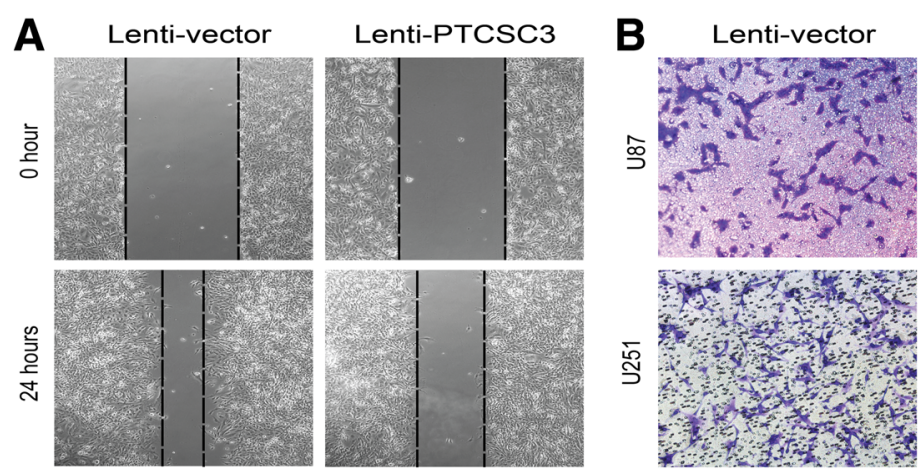

Lenti-PTCSC3
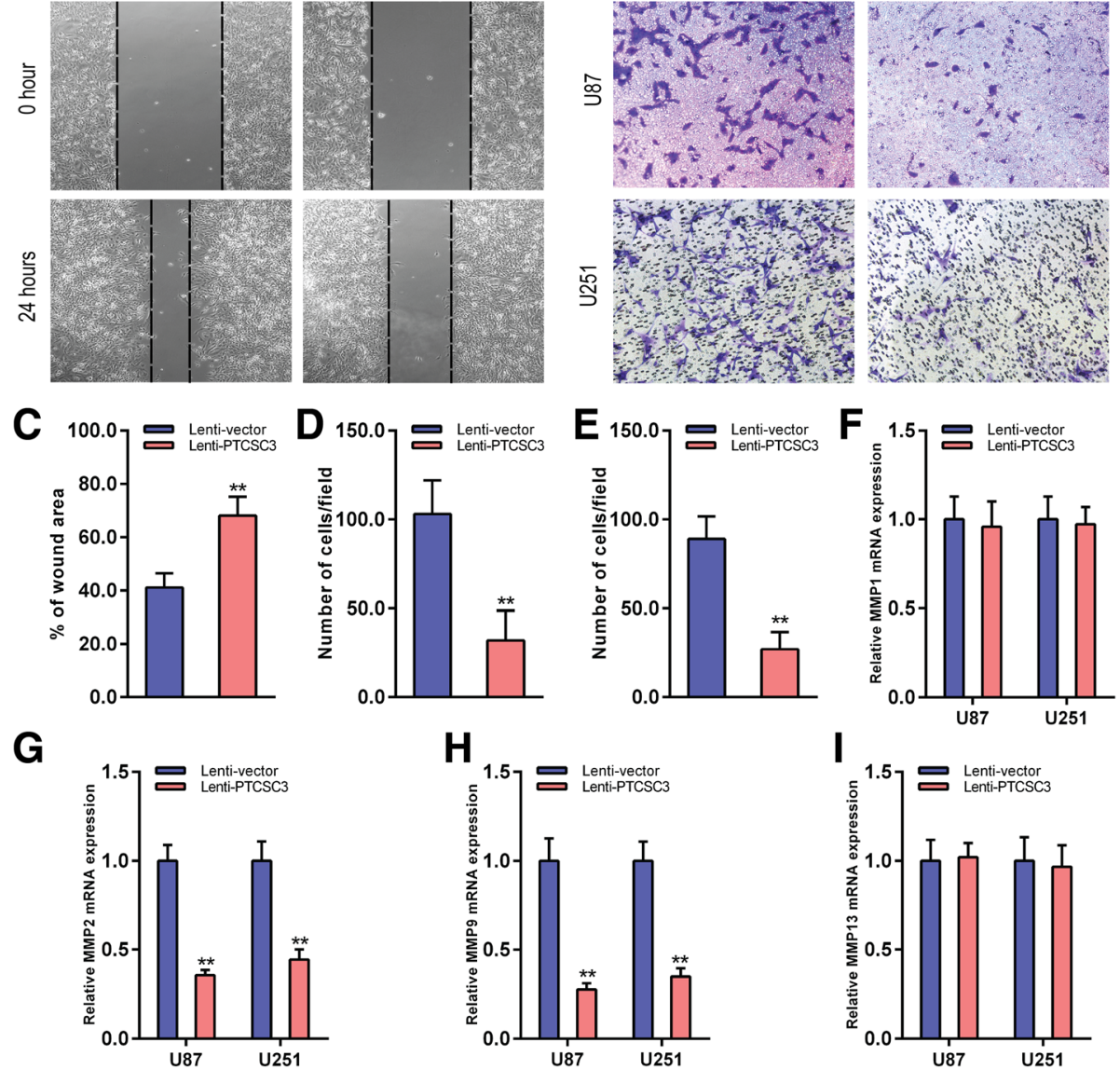

Fig. 4 LnCRNA PTCSC3 overexpression inhibits the migration and invasion of U87 cells. a Wound healing assay was used to determine the migration ability of U87 cells with or without IncRNA PTCSC3 overexpression. Representative images of $0 \mathrm{~h}$ and $24 \mathrm{~h}$ of three repeated experiments are presented. b Transwell assay was performed to determine the invasion ability of U87 and U251 cells with or without IncRNA PTCSC3 overexpression. The representative images of invasive cells in the lower chamber stained with crystal violet. c The quantification of migration ability of U87 cells were presented as percentage of the wound area. The quantification of U87 (d) and U251 (e) cell invasion is presented as invasive cell numbers per field. qRT-PCR analysis of the mRNA expression levels of MMP1 (f), MMP2 (g), MMP9 (h) and MMP13 (i) in U87 and U251 cells with or without InCRNA PTCSC3 overexpression. Data are expressed as the means \pm S.D of three independent experiments. "**" indicates $P<0.01$

\section{Discussion}

It is well-known that lncRNAs are dysregulated in a variety of tumors and play important roles in carcinogenesis as well as cancer progression [11]. In the present study, we detected the expression level of lncRNA PTCSC3 in glioma. We demonstrated that IncRNA PTCSC3 was downregulated in glioma cells compared with normal brain cells. To investigate the biological function of lncRNA PTCSC3, we performed gain-of-function experiments in glioma cell lines. These experiments indicate that overexpression of IncRNA PTCSC3 inhibited proliferation, migration and invasion in glioma cells. For the mechanism study, we explored the influences of dysregulated lncRNA PTCSC3 in the EMT and Wnt/ $\beta$-catenin signaling pathways, revealing a negatively regulatory mechanism between these pathways.
Evidences are accumulating that lncRNAs play pivotal roles in various maligancies. LncRNA H19 was reported to be overexpressed in pancreatic ductal adenocarcinoma and plays oncogenic role through promoting cancer cell proliferation [12]. It was also recognized as a novel biomarker for diagnosis of gastric cancer in plasma [13]. Urothelial Carcinoma Associated 1 (UCA1), which acts as oncogenic lncRNA and promotes bladder cancer progression [14], has been studied in a meta-analysis that up-regulated UCA1 was significantly corrected with LNM and poor OS in patients with various cancers [15]. In glioma, the exploration of lncRNAs is still superficial. LncRNA-ATB was abnormally upregulated in glioma and played an oncogenic role in glioma cells by inhibiting miR-200a and facilitating TGF- $\beta 2$ [16]. LncRNA HULC, which was highly upregulated in liver cancer, 

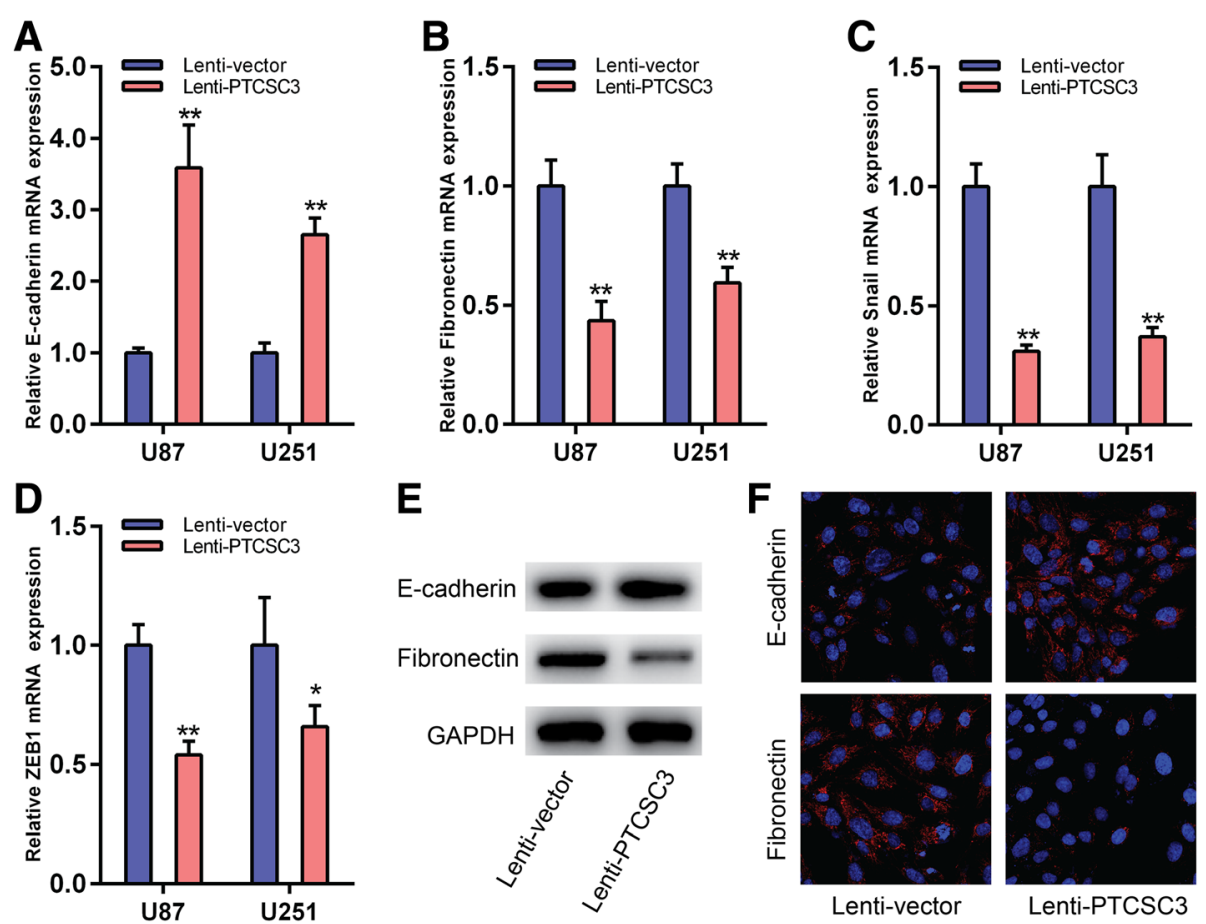

Fig. 5 LnCRNA PTCSC3 overexpression inhibits the epithelial-mesenchymal transition of U87 cells. qRT-PCR analysis of the mRNA expression levels of E-cadherin (a), Fibronectin (b), Snail (c) and ZEB1 (d) in U87 and U251 cells with or without IncRNA PTCSC3 overexpression. e Western blot analysis of E-cadherin and Fibronectin in U87 cells. GAPDH was used as a loading control. Representative images of three repeated experiments are presented. f Representative images of immunofluorenscence analysis of E-cadherin and Fibronectin in U87 cells. Original magnification $400 \times$. Data are expressed as the means \pm S.D of three independent experiments. " ${ }^{\prime *}$ indicates $P<0.05$, " ${ }^{\prime \prime * * " ~ i n d i c a t e s ~} P<0.01$

was discovered as an oncogene in glioma [17]. Another lncRNA MALAT1 (metastasis-associated lung adenocarcinoma transcript 1), which was initially found to be highly expressed in lung cancer and was a favorable prognostic factor for the survival of patients with stage I non-small-cell lung cancer (NSCLC), was demonstrated to have a tumor suppressive role in glioma by attenuating ERK/MAPK-mediated growth and MMP2-mediated invasiveness [18]. This is the first time that lncRNA PTCSC3 was reported in glioma. LncRNA PTCSC3 initially demonstrated that it was a susceptible gene in papillary thyroid carcinoma and that is also maintains the role of inhibition in thyroid cancer invasion [9, 10]. Our results showed that IncRNA PTCSC3 was downregulated in glioma cells. The analysis of tumor cell proliferation and invasion abilities in IncRNA PTCSC3-elevated cells showed the inhibitory role of lncRNA PTCSC3 in glioma, which is similar to its role in thyroid cancer.

To elucidate the possible mechanism by which lncRNA PTCSC3 regulates the proliferation and invasion of glioma cell, qRT-PCR and western blot analysis of the key molecular factors of EMT-associated biomarkers, including E-cadherin, fibronectin, Snail and ZEB1, were assessed in our study. EMT is a process involved in embryonic development, during which cells lose their epithelial features and adopt characteristics of mesenchymal cells. The EMT also plays a role in cancer invasion and metastasis in a sequence of discrete steps $[19,20]$. Decreased expression of the epithelial marker E-cadherin and increased expression of mesenchymal markers, such as $\mathrm{N}$-cadherin and fibronectin, are the most important features of EMT [21]. Transcriptional repressors Snail and ZEB have critical roles in regulating the EMT [22-24]. The activation of these transcriptional factors results in induction of the EMT phenotype [25]. Among the EMT markers we evaluated, the overexpression of lncRNA PTCSC3 led to the downregulation of E-cadherin and upregulation of Fibronectin, Snail and ZEB1, indicating that overexpression of lncRNA PTCSC3 represses the EMT.

EMT activation is induced by a variety of signaling pathways [26-28]. Here, we investigated the Wnt/ $\beta$ catenin signaling pathway in glioma cell lines. Wnt signals are transduced across the membrane by lowdensity lipoprotein receptor-related protein (LRP) receptors [29]. According to our result, LRP6 was inactivated when lncRNA PTCSC3 was overexpressed. The APC/ Axin/GSK/ $\beta$-catenin-complex has been called a "destruction complex" in Wnt signaling [30]. In normal conditions, the complex remains stable. When Axin dissociates, $\beta$-catenin translocates to the nucleus and activates the 
A

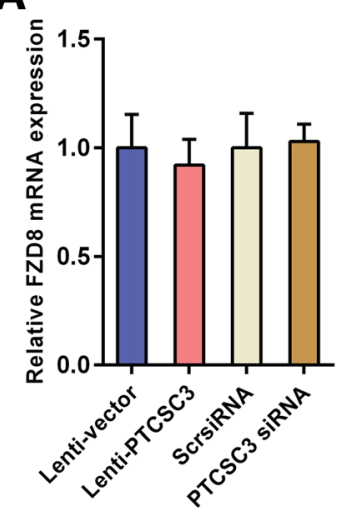

D

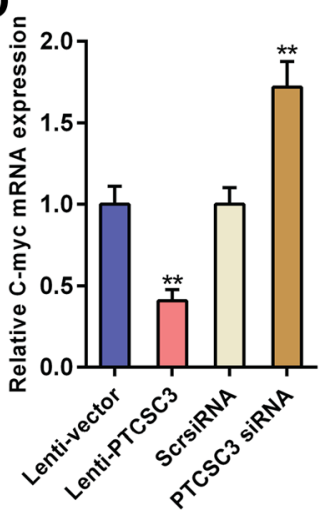

B

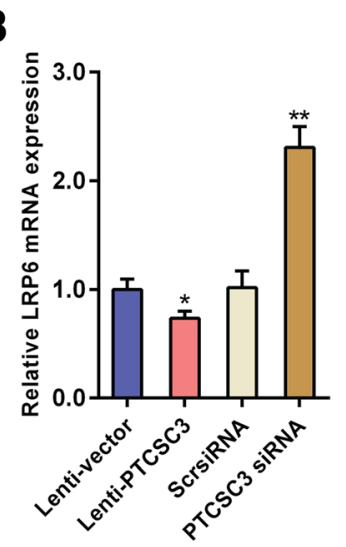

E

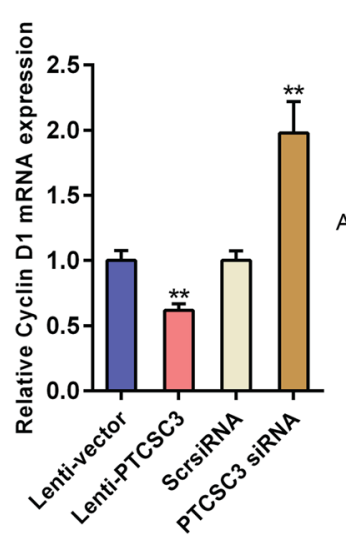

C

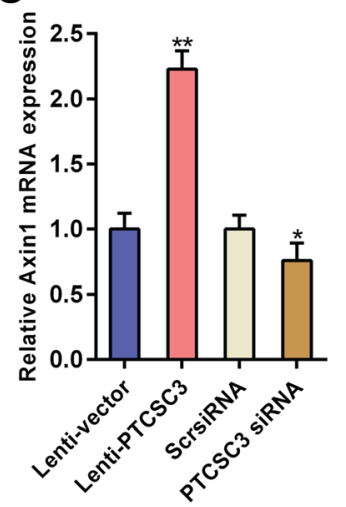

F

Fig. 6 LnCRNA PTCSC3 overexpression inhibits the Wnt/ $\beta$-catenin signaling pathway by suppressing LRP6. qRT-PCR analysis of the mRNA expression levels of FZD8 (a), LRP6 (b), Axin1 (c), C-myc (d) and Cyclin D1 (e) in cells with overexpression or knockdown of IncRNA PTCSC3. f Western blot analysis of LRP6, Axin1, Active catenin, C-myc and Cyclin D1 in U87 cells. GAPDH was used as loading control. Representative images of three repeated experiments are presented. Data are expressed as the means \pm S.D of three independent experiments. " ${ }^{* * \prime}$ indicates $P<0.05$, "***" indicates $P<0.01$

transcription of specific target genes, such as c-Myc and cyclin D1 [31]. Previous reports indicate that the separation of the "destruction complex" leads to an increase in Axin1 protein expression and a concomitant decrease in $\beta$-catenin the cellular environment [32]. Our data revealed results similar to previous studies indicating that dissociation of the complex resulted in an increase in Axin 1 and a reduction in active $\beta$-catenin as well as the subsequent inactivation of c-Myc and cyclin D1. The dissociation of the "destruction complex" was initiated by the inactivation of LRP6, which was primarily controlled by the overexpression of lncRNA PTCSC3.

Matrix metalloproteinases (MMPs) digest extracellular substrates and promote invasion of blood vessels and lymphatics in the spread of cancers [33]. MMP2 and MMP9 are induced by the EMT pathway and promote post-EMT invasion of underlying tissues [21]. Another study also generalized that MMP-2 and MMP-9 are activated by Snail [34]. Moreover, MMPs degrade E-cadherin, disrupting adhesive junctions, to facilitate the EMT [35]. Unsurprisingly, this study showed that MMP2 and MMP9 expression was downregulated in lncRNA PTCSC3overexpressing cells, concomitant with the elevated expression of E-cadherin.

Our study illuminated a complicated mechanism network of how lncRNA PTCSC3 regulated glioma progression. As it was indicated, Wnt/ $\beta$-catenin signaling pathway was initiated by LRP6, which was the target of lncRNA PTCSC3; the "destruction complex" was separated, resulting in a series of downstream alterations (Axin1, C-myc, Cyclin D1, etc.). The activation of Wnt/ $\beta$-catenin signaling pathway induced EMT activation, which could also be regulated by Snail and ZEB1. The expression of MMP2 and MMP9 were downregulated, which was induced by the activation of EMT. In return, the decreased expression of MMP2 and MMP9 could also lead to an accumulation of E-cadherin.

\section{Conclusion}

In conclusion, lncRNA PTCSC3 is downregulated in glioma cells. The overexpression of lncRNA PTCSC3 inhibits proliferation, migration and invasion of glioma 
cells and suppresses the Wnt/ $\mathrm{W}$-catenin signaling pathway through targeting LRP6. LncRNA PTCSC3 is a potential novel therapeutic target for intervention of glioma.

\section{Additional files}

Additional file 1: Figure S1. GRT-PCR analysis of IncRNA PTCSC3 expression levels in U87 transfected with ScrsiRNA or PTCSC3 siRNA. Data are expressed as the means \pm S.D of three independent experiments. " indicates $P<0.01$. (TIF $40 \mathrm{~kb}$ )

Additional file 2: Figure S2. The protein expression level of E-cadherin and Fibronectin in U87 cells with or without InCRNA PTCSC3 overexpression was quantified. Data are expressed as the means \pm S.D of three independent experiments. "***" indicates $P<0.01$. (TIF $47 \mathrm{~kb}$ )

Additional file 3: Figure S3. The protein expression levels of LRP6 (A), Axin 1 (B), Active catenin (C), C-myc (D) and Cyclin D1 in cells with overexpression or knockdown of InCRNA PTCSC3. Data are expressed as the means \pm S.D of three independent experiments. "**" indicates $P<0.01$. (TIF $749 \mathrm{~kb}$ )

\section{Abbreviations}

CCK-8: Cell counting kit-8; DMEM: Dulbecco's Modified Eagle Medium; EMT: Epithelial-mesenchymal transition; FBS: Fetal bovine serum; FZDI: Frizzled class receptor 8; GAPDH: Glyceraldehyde-3-phosphate dehydrogenase; LncRNA PTCSC3: Long noncoding RNA papillary thyroid carcinoma susceptibility candidate 3; LRP6: Low-density lipoprotein receptor-related protein 6; MMP: Matrix metalloproteinase; GRT-PCR: Quantitative reverse transcriptasepolymerase chain reaction; ZEB1: Zinc finger E-box binding homeobox 1

\section{Acknowledgements}

Not applicable.

\section{Funding}

National Natural Science Foundation of China (81471668 \& 81671688). The funder had no role in study design, data analysis, decision to publish, and preparation of the manuscript.

\section{Availability of data and materials}

All data generated or analysed during this study are included in this published article.

\section{Authors' contributions}

SX and RJ performed the experiments. SX created the table and figures. SX wrote the primary manuscript. SX and WZ designed the experiments and modified the paper. WZ supervised the project. WZ provided funding for the entire project. All authors read and approved the final manuscript.

\section{Competing interests}

The authors declare that they have no competing interests.

\section{Consent for publication}

Not applicable.

\section{Ethics approval and consent to participate}

Not applicable.

Received: 25 August 2016 Accepted: 3 February 2017

Published online: 10 February 2017

\section{References}

1. Caruso G, Caffo M. Antisense oligonucleotides in the treatment of cerebral gliomas. Review of concerning patents. Recent Pat CNS Drug Discov. 2014; 9(1):2-12.

2. Taylor LP. Diagnosis, treatment, and prognosis of glioma: five new things. Neurology. 2010;75(18 Suppl 1):S28-32.

3. Omuro A, DeAngelis LM. Glioblastoma and other malignant gliomas: a clinical review. JAMA. 2013;310(17):1842-50.
4. Van Meir EG, Hadjipanayis CG, Norden AD, et al. Exciting new advances in neuro-oncology: the avenue to a cure for malignant glioma. CA Cancer J Clin. 2010;60(3):166-93.

5. Wen PY, Kesari S. Malignant gliomas in adults. N Engl J Med. 2008;359(5): 492-507.

6. Struhl K. Transcriptional noise and the fidelity of initiation by RNA polymerase II. Nat Struct Mol Biol. 2007;14(2):103-5.

7. Gupta RA, Shah N, Wang KC, et al. Long non-coding RNA HOTAIR reprograms chromatin state to promote cancer metastasis. Nature. 2010; 464(7291):1071-6.

8. Jendrzejewski J, He H, Radomska HS, et al. The polymorphism rs944289 predisposes to papillary thyroid carcinoma through a large intergenic noncoding RNA gene of tumor suppressor type. Proc Natl Acad Sci U S A. 2012;109(22):8646-51.

9. Fan M, Li X, Jiang W, et al. A long non-coding RNA, PTCSC3, as a tumor suppressor and a target of miRNAs in thyroid cancer cells. Exp Ther Med. 2013;5(4):1143-6.

10. Jendrzejewski J, Thomas A, Liyanarachchi S, et al. PTCSC3 Is Involved in Papillary Thyroid Carcinoma Development by Modulating S100A4 Gene Expression. J Clin Endocrinol Metab. 2015;100(10):E1370-7.

11. Huarte M. The emerging role of IncRNAs in cancer. Nat Med. 2015;21(11): $1253-61$.

12. Ma $L$, Tian $X$, Wang $F$, et al. The long noncoding RNA H19 promotes cell proliferation via E2F-1 in pancreatic ductal adenocarcinoma. Cancer Biol Ther. 2016;17(10):1051-61.

13. Zhou X, Yin C, Dang Y, et al. Identification of the long non-coding RNA H19 in plasma as a novel biomarker for diagnosis of gastric cancer. Sci Rep. 2015:5:11516.

14. Xue M, Chen W, Li X. Urothelial cancer associated 1: a long noncoding RNA with a crucial role in cancer. J Cancer Res Clin Oncol. 2016;142(7):1407-19.

15. He A, Hu R, Chen Z, et al. Role of long noncoding RNA UCA1 as a common molecular marker for lymph node metastasis and prognosis in various cancers: a meta-analysis. Oncotarget. 2016.

16. Ma CC, Xiong Z, Zhu GN, et al. Long non-coding RNA ATB promotes glioma malignancy by negatively regulating miR-200a. J Exp Clin Cancer Res. 2016;35(1):90.

17. Zhu Y, Zhang X, Qi L, et al. HULC long noncoding RNA silencing suppresses angiogenesis by regulating ESM-1 via the PI3K/Akt/mTOR signaling pathway in human gliomas. Oncotarget. 2016;7(12):14429-40.

18. Han $Y$, Wu Z, Wu T, et al. Tumor-suppressive function of long noncoding RNA MALAT1 in glioma cells by downregulation of MMP2 and inactivation of ERK/MAPK signaling. Cell Death Dis. 2016;7, e2123.

19. Hanahan D, Weinberg RA. Hallmarks of cancer: the next generation. Cell. 2011;144(5):646-74.

20. Thiery JP. Epithelial-mesenchymal transitions in tumour progression. Nat Rev Cancer. 2002;2(6):442-54.

21. Kalluri R, Weinberg RA. The basics of epithelial-mesenchymal transition. J Clin Invest. 2009;119(6):1420-8.

22. Cano A, Perez-Moreno MA, Rodrigo I, et al. The transcription factor snail controls epithelial-mesenchymal transitions by repressing E-cadherin expression. Nat Cell Biol. 2000;2(2):76-83.

23. Vandewalle C, Comijn J, De Craene B, et al. SIP1/ZEB2 induces EMT by repressing genes of different epithelial cell-cell junctions. Nucleic Acids Res. 2005;33(20):6566-78

24. Bracken CP, Gregory PA, Kolesnikoff N, et al. A double-negative feedback loop between ZEB1-SIP1 and the microRNA-200 family regulates epithelialmesenchymal transition. Cancer Res. 2008;68(19):7846-54.

25. Davalos V, Moutinho C, Villanueva A, et al. Dynamic epigenetic regulation of the microRNA-200 family mediates epithelial and mesenchymal transitions in human tumorigenesis. Oncogene. 2012;31(16):2062-74.

26. Taipale J, Beachy PA. The Hedgehog and Wnt signalling pathways in cancer. Nature. 2001;411(6835):349-54.

27. Espinoza I, Miele L. Deadly crosstalk: Notch signaling at the intersection of EMT and cancer stem cells. Cancer Lett. 2013:341(1):41-5.

28. Katoh Y, Katoh M. FGFR2-related pathogenesis and FGFR2-targeted therapeutics (Review). Int J Mol Med. 2009;23(3):307-11.

29. MacDonald BT, Tamai K, He X. Wnt/beta-catenin signaling: components, mechanisms, and diseases. Dev Cell. 2009;17(1):9-26.

30. Behrens J, Jerchow BA, Wurtele $M$, et al. Functional interaction of an axin homolog, conductin, with beta-catenin, APC, and GSK3beta. Science. 1998; 280(5363):596-9. 
31. Gehrke I, Gandhirajan RK, Kreuzer KA. Targeting the WNT/beta-catenin/TCF/ LEF1 axis in solid and haematological cancers: Multiplicity of therapeutic options. Eur J Cancer. 2009;45(16):2759-67.

32. Schmitz $Y$, Rateitschak $\mathrm{K}$, Wolkenhauer $\mathrm{O}$. Analysing the impact of nucleocytoplasmic shuttling of beta-catenin and its antagonists APC, Axin and GSK3 on Wnt/beta-catenin signalling. Cell Signal. 2013;25(11):2210-21.

33. Willis AL, Sabeh F, Li XY, et al. Extracellular matrix determinants and the regulation of cancer cell invasion stratagems. J Microsc. 2013;251(3):250-60.

34. Qiao B, Johnson NW, Gao J. Epithelial-mesenchymal transition in oral squamous cell carcinoma triggered by transforming growth factor-beta1 is Snail family-dependent and correlates with matrix metalloproteinase-2 and -9 expressions. Int J Oncol. 2010;37(3):663-8.

35. Nistico P, Bissell MJ, Radisky DC. Epithelial-mesenchymal transition: general principles and pathological relevance with special emphasis on the role of matrix metalloproteinases. Cold Spring Harb Perspect Biol. 2012;4:2.

Submit your next manuscript to BioMed Central and we will help you at every step:

- We accept pre-submission inquiries

- Our selector tool helps you to find the most relevant journal

- We provide round the clock customer support

- Convenient online submission

- Thorough peer review

- Inclusion in PubMed and all major indexing services

- Maximum visibility for your research

Submit your manuscript at www.biomedcentral.com/submit
Biomed Central 